\title{
General Asymptotic Supnorm Estimates for Solutions of One-Dimensional Advection-Diffusion Equations in Heterogeneous Media
}

\author{
José A. Barrionuevo, Lucas S. Oliveira, and Paulo R. Zingano \\ Departamento de Matemática Pura e Aplicada, Universidade Federal do Rio Grande do Sul, 91509-900 Porto Alegre, RS, Brazil \\ Correspondence should be addressed to Paulo R. Zingano; zingano@gmail.com
}

Received 18 October 2013; Accepted 1 March 2014; Published 8 May 2014

Academic Editor: Chi K. Lin

Copyright (C) 2014 José A. Barrionuevo et al. This is an open access article distributed under the Creative Commons Attribution License, which permits unrestricted use, distribution, and reproduction in any medium, provided the original work is properly cited.

We derive general bounds for the large time size of supnorm values $\|u(\cdot, t)\|_{L^{\infty}(\mathbb{R})}$ of solutions to one-dimensional advectiondiffusion equations $u_{t}+(b(x, t) u)_{x}=u_{x x}, x \in \mathbb{R}, t>0$ with initial data $u(\cdot, 0) \in L^{p_{0}}(\mathbb{R}) \cap L^{\infty}(\mathbb{R})$ for some $1 \leq p_{0}<\infty$ and arbitrary bounded advection speeds $b(x, t)$, introducing new techniques based on suitable energy arguments. Some open problems and related results are also given.

\section{Introduction}

In this work, we obtain very general large time estimates for supnorm values of solutions $u(\cdot, t)$ to parabolic initial value problems of the form

$$
\begin{gathered}
u_{t}+(b(x, t) u)_{x}=u_{x x}, \quad x \in \mathbb{R}, t>0, \\
u(\cdot, 0)=u_{0} \in L^{p_{0}}(\mathbb{R}) \cap L^{\infty}(\mathbb{R}), \quad 1 \leq p_{0}<\infty,
\end{gathered}
$$

for arbitrary continuously differentiable advection fields $b \in$ $L^{\infty}(\mathbb{R} \times[0, \infty[)$. Here, by solution to (la) and (lb) in some time interval $\left[0, T_{*}\left[, 0<T_{*} \leq \infty\right.\right.$, we mean a function $u: \mathbb{R} \times$ $\left[0, T_{*}\left[\rightarrow \mathbb{R}\right.\right.$ which is bounded in each strip $S_{T}=\mathbb{R} \times[0, T]$, $0<T<T_{*}$, solves (1a) in the classical sense for $0<t<T_{*}$, and satisfies $u(\cdot, t) \rightarrow u_{0}$ in $L_{\text {loc }}^{1}(\mathbb{R})$ as $t \rightarrow 0$. It follows from the a priori estimates given in Section 2 that all solutions of problem (1a), (1b) are actually globally defined $\left(T_{*}=\infty\right)$, with $u(\cdot, t) \in C^{0}\left(\left[0, \infty\left[, L^{p}(\mathbb{R})\right)\right.\right.$ for each $p \geq p_{0}$ finite. Given $b \in L^{\infty}(\mathbb{R} \times[0, \infty[)$, what then can be said about the size of supnorm values $\|u(\cdot, t)\|_{L^{\infty}(\mathbb{R})}$ for $t \gg 1$ ?
When $\partial b / \partial x \geq 0$ for all $x \in \mathbb{R}, t \geq 0$, it is well known that, for each $p_{0} \leq p \leq \infty,\|u(\cdot, t)\|_{L^{p}(\mathbb{R})}$ is monotonically decreasing in $t$, with

$$
\|u(\cdot, t)\|_{L^{\infty}(\mathbb{R})} \leq K\left(p_{0}\right)\left\|u_{0}\right\|_{L^{p_{0}(\mathbb{R})}} t^{-1 / 2 p_{0}} \quad \forall t>0 \quad\left(b_{x} \geq 0\right)
$$

for some constant $0<K\left(p_{0}\right)<2^{-1 / p_{0}}$ that depends only on $p_{0}$; see, for example, [1-5]. For general $b(x, t)$, however, estimating $\|u(\cdot, t)\|_{L^{\infty}(\mathbb{R})}$ is much harder. To see why, let us illustrate with the important case $p_{0}=1$, where one has

$$
\|u(\cdot, t)\|_{L^{1}(\mathbb{R})} \leq\left\|u_{0}\right\|_{L^{1}(\mathbb{R})} \quad \forall t>0,
$$

as recalled in Theorem 1 . Writing (1a) as

$$
u_{t}+b(x, t) u_{x}=u_{x x}-b_{x}(x, t) u,
$$

we observe on the right hand side of (4) that $|u(x, t)|$ is pushed to grow at points $(x, t)$ where $b_{x}(x, t)<0$. If this condition persists long enough, large values of $|u(x, t)|$ might be generated, particularly at sites where $-b_{x}(x, t) \gg 1$. Now, because of constraint (3), any persistent growth in solution size will eventually create long thin structures as shown in 


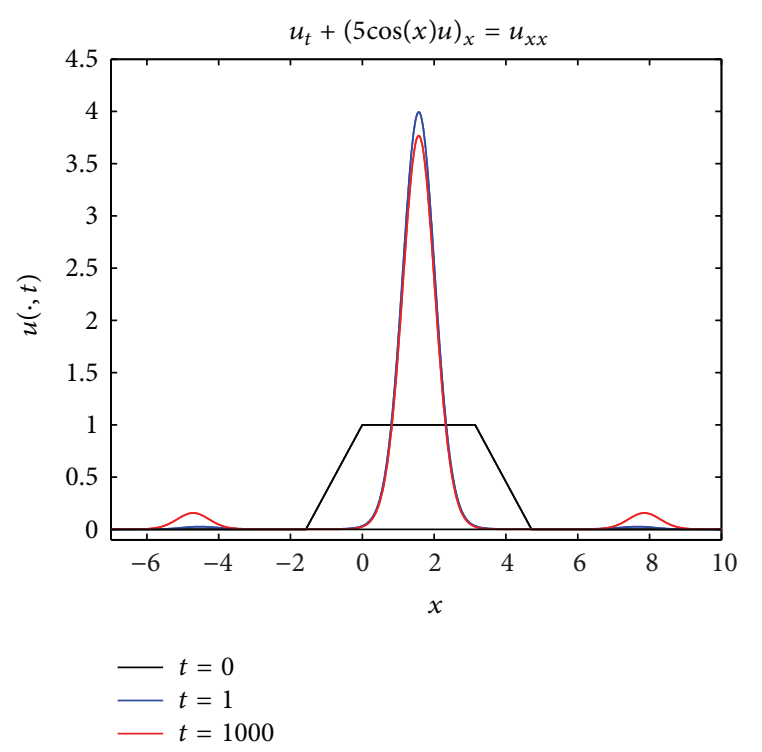

FIGURE 1: Solution profiles showing typical growth in regions with $b_{x}<0$, where $b=5 \cos x$. After reaching maximum height, solution starts decaying very slowly due to its spreading and mass conservation (decay rate is not presently known).

Figure 1, which, in turn, tend to be effectively dissipated by viscosity. The final overall behavior that ultimately results from such competition is not immediately clear, either on physical or on mathematical grounds.

As shown in (4), it is not the magnitude of $b(x, t)$ itself but instead its oscillation that is relevant in determining $\|u(\cdot, t)\|_{L^{\infty}(\mathbb{R})}$. Accordingly, we introduce the quantity $B(t)$ defined by

$$
B(t)=\frac{1}{2}\left(\sup _{x \in \mathbb{R}} b(x, t)-\inf _{x \in \mathbb{R}} b(x, t)\right), \quad t \geq 0,
$$

which plays a fundamental role in the analysis. Our main result is now easily stated.

Main Theorem. For each $p \geq p_{0}$, one has ${ }^{1}$

$$
\begin{aligned}
& \limsup _{t \rightarrow \infty}\|u(\cdot, t)\|_{L^{\infty}(\mathbb{R})} \\
& \quad \leq\left(\frac{3 \sqrt{3}}{2 \pi} p\right)^{1 / p} \cdot \mathscr{B}^{1 / p} \cdot \limsup _{t \rightarrow \infty}\|u(\cdot, t)\|_{L^{p}(\mathbb{R})},
\end{aligned}
$$

where $\mathscr{B}=\lim \sup _{t \rightarrow \infty} B(t)$.

In particular, in the important case $p_{0}=1$ considered above, we obtain, using (3),

$$
\limsup _{t \rightarrow \infty}\|u(\cdot, t)\|_{L^{\infty}(\mathbb{R})} \leq\left(\frac{3 \sqrt{3}}{2 \pi}\right) \cdot \mathscr{B} \cdot\left\|u_{0}\right\|_{L^{1}(\mathbb{R})},
$$

so that $u(\cdot, t)$ stays uniformly bounded for all time in this case. ${ }^{2}$ Estimates similar to (6) can also be shown to hold for the $n$-dimensional problem

$$
u_{t}+\operatorname{div}(\mathbf{b}(x, t) u)=\Delta u, \quad u(\cdot, 0) \in L^{p}\left(\mathbb{R}^{n}\right) \cap L^{\infty}\left(\mathbb{R}^{n}\right),
$$

but to simplify our discussion we consider here the case $n=1$ only. Our derivation of (6), which improves some unpublished results by the third author, uses the 1D inequality

$$
\|\mathrm{v}\|_{L^{\infty}(\mathbb{R})} \leq C_{\infty}\|\mathrm{v}\|_{L^{1}(\mathbb{R})}^{1 / 3}\left\|\mathrm{v}_{x}\right\|_{L^{2}(\mathbb{R})}^{2 / 3}, \quad \mathrm{v} \in L^{1}(\mathbb{R}) \cap H^{1}(\mathbb{R}),
$$

where $C_{\infty}=(3 / 4)^{2 / 3}$, and can be readily extended to other problems of interest like $1 \mathrm{D}$ systems of viscous conservation laws [6, Ch. 9] or the more general equation

$$
\begin{gathered}
u_{t}+(b(x, t, u) u)_{x}=\left(a(x, t, u) u_{x}\right)_{x}, \\
a(x, t, u) \geq \mu(t)>0,
\end{gathered}
$$

with bounded values $b(x, t, u)$; provided that we assume $\int^{\infty} \mu(t) d t=\infty$ : using a similar argument, we get the estimate $^{3}[7$, Ch. 2]

$$
\begin{aligned}
& \limsup _{t \rightarrow \infty}\|u(\cdot, t)\|_{L^{\infty}(\mathbb{R})} \\
& \quad \leq\left(\frac{3 \sqrt{3}}{2 \pi} p\right)^{1 / p} \cdot \mathscr{B}_{\mu}^{1 / p} \cdot \limsup _{t \rightarrow \infty}\|u(\cdot, t)\|_{L^{p}(\mathbb{R})},
\end{aligned}
$$

for each $p \geq p_{0}$, where

$$
\begin{gathered}
\mathscr{B}_{\mu}=\limsup _{t \rightarrow \infty} \frac{B(t)}{\mu(t)}, \\
B(t)=\frac{1}{2}\left(\sup _{x \in \mathbb{R}} b(x, t, u(x, t))-\inf _{x \in \mathbb{R}} b(x, t, u(x, t))\right) .
\end{gathered}
$$

More involving applications, such as problems with superlinear advection or degenerate diffusion, which require considerable extra work, will be studied in the future.

\section{A Priori Estimates}

This section contains some preliminary results on the solutions of problem (1a) and (1b) needed later for our derivation of estimate (6), which is completed in Section 3. (Recall that a solution on some given time interval $\left[0, T_{*}\left[, 0<T_{*} \leq\right.\right.$ $\infty$, is a function $u(\cdot, t) \in L_{\text {loc }}^{\infty}\left(\left[0, T_{*}\left[, L^{\infty}(\mathbb{R})\right)\right.\right.$ which is smooth $\left(C^{2}\right.$ in $x, C^{1}$ in $\left.t\right)$ in $\left.\mathbb{R} \times\right] 0, T_{*}$ [ and solves (1a) there, verifying the initial condition in the sense of $L_{\text {loc }}^{1}(\mathbb{R})$, i.e., $\left\|u(\cdot, t)-u_{0}\right\|_{L^{1}(\mathbb{K})} \rightarrow 0$ as $t \rightarrow 0$ for each compact $\mathbb{K} \subset \mathbb{R}$. Local existence theory can be found in, e.g., [8, Ch. 6].) We start with a simple Gronwall-type estimate for $\|u(\cdot, t)\|_{L^{q}(\mathbb{R})}$, $p_{0} \leq q<\infty$. The corresponding result for the supnorm $(q=\infty)$ is more difficult to obtain and will be given at the end of Section 2; see Theorem 4.

Theorem 1. If $u(\cdot, t) \in L_{\mathrm{loc}}^{\infty}\left(\left[0, T_{*}\left[, L^{\infty}(\mathbb{R})\right)\right.\right.$ solves problem (1a), (1b), then $u(\cdot, t) \in C^{0}\left(\left[0, T_{*}\left[, L^{q}(\mathbb{R})\right)\right.\right.$ for each $p_{0} \leq q<$ $\infty$, and

$$
\begin{aligned}
& \|u(\cdot, t)\|_{L^{q}(\mathbb{R})} \\
& \quad \leq\|u(\cdot, 0)\|_{L^{q}(\mathbb{R})} \cdot \exp \left\{\frac{1}{2}(q-1) \int_{0}^{t} B(\tau)^{2} d \tau\right\}
\end{aligned}
$$

for all $0<t<T_{*}$. 
Proof. The proof is standard, so we will only sketch the basic steps. Taking $S \in C^{1}(\mathbb{R})$ such that $S^{\prime}(\mathrm{v}) \geq 0$ for all v, $S(0)=0, S(\mathrm{v})=\operatorname{sgn}(\mathrm{v})$ for $|\mathrm{v}| \geq 1$, let (given $\delta>$ 0) $L_{\delta}(\mathrm{u})=\int_{0}^{\mathrm{u}} S(\mathrm{v} / \delta) d \mathrm{v}$, so that $L_{\delta}(\mathrm{u}) \rightarrow|\mathrm{u}|$ as $\delta \rightarrow 0$, uniformly in $\mathrm{u}$. Let $\Phi_{\delta}(\mathrm{u})=L_{\delta}(\mathrm{u})^{q}$. Given $R>0,0<\epsilon \leq 1$, let $\zeta_{R}(\cdot)$ be the cut-off function $\zeta_{R}(x)=0$ for $|x| \geq R, \zeta_{R}(x)=$ $\exp \left\{-\epsilon \sqrt{1+x^{2}}\right\}-\exp \left\{-\epsilon \sqrt{1+R^{2}}\right\}$ for $|x|<R$. Multiplying (1a) by $\Phi_{\delta}^{\prime}(u(x, t)) \cdot \zeta_{R}(x)$ if $q \neq 2$, or $u(x, t) \cdot \zeta_{R}(x)$ if $q=2$, and integrating the result on $\mathbb{R} \times[0, t]$, we obtain, letting $\delta \rightarrow 0$ and then $R \rightarrow \infty$, since $u \in L^{\infty}(\mathbb{R} \times[0, t])$,

$$
\begin{gathered}
\mathrm{U}_{\epsilon}(t)+V_{\epsilon}(t) \leq \mathrm{U}_{\epsilon}(0)+\int_{0}^{t} G_{\epsilon}(\tau) \mathrm{U}_{\epsilon}(\tau) d \tau, \\
\mathrm{U}_{\epsilon}(t)=\int_{\mathbb{R}}|u(x, t)|^{q} w_{\epsilon}(x) d x,
\end{gathered}
$$

where $w_{\epsilon}(x)=\exp \left\{-\epsilon \sqrt{1+x^{2}}\right\}, G_{\epsilon}(t)=(1 / 2) q(q-1) B(t)^{2}+$ $\epsilon 2 q \cdot \sup _{0 \leq \tau \leq t}\|u(\cdot, \tau)\|_{L^{\infty}(\mathbb{R})}+\epsilon$, and

$$
\begin{aligned}
& V_{\epsilon}(t)
\end{aligned}
$$

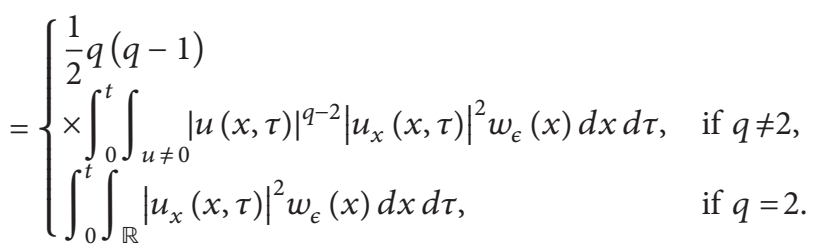

By Gronwall's lemma, (14a) and (14b) give $\mathrm{U}_{\epsilon}(t) \leq$ $\mathrm{U}_{\epsilon}(0) \cdot \exp \left\{\int_{0}^{t} G_{\epsilon}(\tau) d \tau\right\}$, from which we obtain (13) by simply letting $\epsilon \rightarrow 0$. This shows, in particular, that $u(\cdot, t) \in L_{\text {loc }}^{\infty}\left(\left[0, T_{*}\left[, L^{q}(\mathbb{R})\right)\right.\right.$ if $p_{0} \leq q<\infty$. Now, to get $u(\cdot, t) \in C^{0}\left(\left[0, T_{*}\left[, L^{q}(\mathbb{R})\right)\right.\right.$, it is sufficient to show that, given $\varepsilon>0$ and $0<T<T_{*}$ arbitrary, we can find $R=R(\varepsilon, T) \gg 1$ large enough so that we have $\|u(\cdot, t)\|_{\left.L^{q}|x|>R\right)}<\varepsilon$ for any $0 \leq t \leq T$. Taking $\psi \in C^{2}(\mathbb{R})$ with $0 \leq \psi \leq 1$ and $\psi(x)=0$ for all $x \leq 0, \psi(x)=1$ for all $x \geq 1$, let $\Psi_{R, M} \in C^{2}(\mathbb{R})$ be the cut-off function given by $\Psi_{R, M}(x)=0$ if $|x| \leq R-1$, $\Psi_{R, M}(x)=\psi(|x|-R+1)$ if $R-1<|x|<R$, and $\Psi_{R, M}(x)=1$ if $R \leq|x| \leq R+M, \Psi_{R, M}(x)=\psi(R+M+1-|x|)$ if $R+M<|x|<R+M+1, \Psi_{R, M}(x)=0$ if $|x| \geq R+M+1$, where $R>1, M>0$ are given. Multiplying (la) by $\Phi_{\delta}^{\prime}(u(x, t)) \cdot \Psi_{R, M}(x)$ if $q \neq 2$, or $u(x, t) \cdot \Psi_{R, M}(x)$ if $q=2$, and integrating the result on $\mathbb{R} \times[0, t], 0<t \leq T$, we obtain, as in (14a) and (14b), by letting $\delta \rightarrow 0, M \rightarrow \infty$, that $\|u(\cdot, t)\|_{\left.L^{q}|x|>R\right)}<\varepsilon / 2+\|u(\cdot, 0)\|_{\left.L^{q}|| x \mid>R-1\right)}$ for all $0 \leq t \leq T$, provided that we take $R>1$ sufficiently large. This gives the continuity result, and the proof is complete.

An important by-product of the proof above is that we have (letting $\epsilon \rightarrow 0$ in (14a) and (14b), and using (13)), for each $0<T<T_{*}$ and $q \geq \max \left\{p_{0}, 2\right\}$,

$$
\int_{0}^{T} \int_{\mathbb{R}}|u(x, \tau)|^{q-2}\left|u_{x}(x, \tau)\right|^{2} d x d \tau<\infty .
$$

Therefore, if we repeat the steps above leading to (14a) and (14b), we obtain (letting $\delta \rightarrow 0, R \rightarrow \infty, \epsilon \rightarrow 0$, in this order, taking (13) and (15) into account) the identity

$$
\begin{gathered}
\|u(\cdot, t)\|_{L^{q}(\mathbb{R})}^{q}+q(q-1) \int_{0}^{t} \int_{\mathbb{R}}|u(x, \tau)|^{q-2}\left|u_{x}(x, \tau)\right|^{2} d x d \tau \\
=\|u(\cdot, 0)\|_{L^{q}(\mathbb{R})}^{q} \\
+q(q-1) \int_{0}^{t} \int_{\mathbb{R}}(b(x, \tau)-\beta(\tau))|u(x, \tau)|^{q-2} \\
\times u(x, \tau) u_{x}(x, \tau) d x d \tau
\end{gathered}
$$

for every $0<t<T_{*}$ and $\max \left\{p_{0}, 2\right\} \leq q<\infty$, where

$$
\beta(t)=\frac{1}{2}\left(\sup _{x \in \mathbb{R}} b(x, t)+\inf _{x \in \mathbb{R}} b(x, t)\right), \quad t \geq 0 .
$$

The core of the difficulty in the analysis of (la) and (1b) is apparent here: under the sole assumption that $b$ is bounded, it is not much clear how one should go about the last term in (16) in order to get more than (13) above. Actually, it will be convenient to consider (16) in the (equivalent) differential form, that is,

$$
\begin{aligned}
& \frac{d}{d t}\|u(\cdot, t)\|_{L^{q}(\mathbb{R})}^{q} \\
& +q(q-1) \int_{\mathbb{R}}|u(x, t)|^{q-2}\left|u_{x}(x, t)\right|^{2} d x \\
& =q(q-1) \int_{\mathbb{R}}(b(x, t)-\beta(t))|u(x, t)|^{q-2} \\
& \times u(x, t) u_{x}(x, t) d x
\end{aligned}
$$

for all $t \in\left[0, T_{*}\left[\backslash E_{q}\right.\right.$, where $E_{q} \subset\left[0, T_{*}[\right.$ has zero measure. We then readily obtain, using (9) and the one-dimensional Nash inequality [9]

$$
\|\mathrm{v}\|_{L^{2}(\mathbb{R})} \leq C_{2}\|\mathrm{v}\|_{L^{1}(\mathbb{R})}^{2 / 3}\left\|\mathrm{v}_{x}\right\|_{L^{2}(\mathbb{R})}^{1 / 3}, \quad C_{2}=\left(\frac{3 \sqrt{3}}{4 \pi}\right)^{1 / 3},
$$

where the value given above for $C_{2}$ is optimal [10], the following result.

Theorem 2. Let $q \geq 2 p_{0}$. If $\hat{t} \in\left[0, T_{*}\left[\backslash E_{q}\right.\right.$ is such that $(d / d t)\|u(\cdot, t)\|_{\left.L^{q}(\mathbb{R})\right|_{t=\hat{t}}}^{q} \geq 0$, then

$$
\begin{gathered}
\|u(\cdot, \hat{t})\|_{L^{q(\mathbb{R})}} \leq\left(\frac{q}{2} C_{2}^{3}\right)^{1 / q} B(\hat{t})^{1 / q}\|u(\cdot, \hat{t})\|_{L^{q / 2}(\mathbb{R})}, \\
\|u(\cdot, \hat{t})\|_{L^{\infty}(\mathbb{R})} \leq\left(\frac{q}{2} C_{2} C_{\infty}\right)^{2 / q} B(\widehat{t})^{2 / q}\|u(\cdot, \hat{t})\|_{L^{q / 2}(\mathbb{R})} .
\end{gathered}
$$

Proof. Consider (20a) first. From (5), (17), and (18), we have

$$
\begin{aligned}
& \int_{\mathbb{R}}|u(x, \widehat{t})|^{q-2}\left|u_{x}(x, \widehat{t})\right|^{2} d x \\
& \quad \leq B(\widehat{t}) \int_{\mathbb{R}}|u(x, \widehat{t})|^{q-1}\left|u_{x}(x, \widehat{t})\right| d x .
\end{aligned}
$$


This gives

$$
\int_{\mathbb{R}}|u(x, \widehat{t})|^{q-2}\left|u_{x}(x, \widehat{t})\right|^{2} d x \leq B(\widehat{t})^{2}\|u(\cdot, \widehat{t})\|_{L^{q}(\mathbb{R})}^{q},
$$

or, in terms of $\widehat{v} \in L^{1}(\mathbb{R}) \cap L^{\infty}(\mathbb{R})$ defined by $\widehat{v}(x)=$ $|u(x, \widehat{t})|^{q / 2}$ if $q>2, \widehat{v}(x)=u(x, \widehat{t})$ if $q=2$,

$$
\left\|\widehat{v}_{x}\right\|_{L^{2}(\mathbb{R})} \leq \frac{q}{2} B(\widehat{t})\|\widehat{v}\|_{L^{2}(\mathbb{R})} .
$$

Using (19), we then get $\|\widehat{v}\|_{L^{2}(\mathbb{R})}^{2} \leq(q / 2) C_{2}^{3} B(\widehat{t})\|\widehat{v}\|_{L^{1}(\mathbb{R})}^{2}$, which is equivalent to (20a). Similarly, (20b) can be obtained, using (9).

Thus, we can use (20a) and (20b) when $\|u(\cdot, t)\|_{L^{q}(\mathbb{R})}$ is not decreasing. If it is decreasing, (18) becomes useless but at least we know in such case that $\|u(\cdot, t)\|_{L^{q(\mathbb{R})}}$ is not increasing, which should be useful too. Different values of $q$ have different scenarios, which we will have to piece together in some way. The next result shows us just how. To this end, it is convenient to introduce the quantities $\mathbb{B}\left(t_{0} ; t\right)$ and $\mathbb{U}_{p}\left(t_{0} ; t\right)$ defined by

$$
\begin{gathered}
\mathbb{B}\left(t_{0} ; t\right)=\sup \left\{B(\tau): t_{0} \leq \tau \leq t\right\}, \\
\mathbb{U}_{p}\left(t_{0} ; t\right)=\sup \left\{\|u(\cdot, \tau)\|_{L^{p}(\mathbb{R})}: t_{0} \leq \tau \leq t\right\},
\end{gathered}
$$

given $p \geq p_{0}, 0 \leq t_{0} \leq t<T_{*}$ arbitrary.

Theorem 3. Let $q \geq 2 p_{0}$. For each $0 \leq t_{0}<T_{*}$, we have

$$
\begin{aligned}
& \mathbb{U}_{q}\left(t_{0} ; t\right) \\
& \quad \leq \max \left\{\left\|u\left(\cdot, t_{0}\right)\right\|_{L^{q}(\mathbb{R})} ;\left(\frac{q}{2} C_{2}^{3}\right)^{1 / q} \mathbb{B}\left(t_{0} ; t\right)^{1 / q_{\mathbb{U}_{q / 2}}}\left(t_{0} ; t\right)\right\}
\end{aligned}
$$

for all $t_{0} \leq t<T_{*}$.

Proof. Set $\lambda_{q}(t)=\left((q / 2) C_{2}^{3}\right)^{1 / q} \mathbb{B}\left(t_{0} ; t\right)^{1 / q} \mathbb{U}_{q / 2}\left(t_{0} ; t\right)$. There are three cases to consider.

Case I. $\|u(\cdot, \tau)\|_{L^{q}(\mathbb{R})}>\lambda_{q}(t)$ for all $t_{0} \leq \tau \leq t$. By (20a), Theorem 2, we must then have $(d / d \tau)\|u(\cdot, \tau)\|_{L^{q}(\mathbb{R})}^{q}<0$ for all $\tau \in\left[t_{0}, t\right] \backslash E_{q}$, so that $\|u(\cdot, \tau)\|_{L^{q}(\mathbb{R})}$ is monotonically decreasing in $\left[t_{0}, t\right]$. In particular, $\mathbb{U}_{q}\left(t_{0} ; t\right)=\left\|u\left(\cdot, t_{0}\right)\right\|_{L^{q}(\mathbb{R})}$ in this case, and (26) holds.

Case II. $\left\|u\left(\cdot, t_{0}\right)\right\|_{L^{q}(\mathbb{R})}>\lambda_{q}(t)$ and $\left\|u\left(\cdot, t_{1}\right)\right\|_{L^{q}(\mathbb{R})} \leq \lambda_{q}(t)$ for some $\left.\left.t_{1} \in\right] t_{0}, t\right]$. In this case, let $\left.\left.t_{2} \in\right] t_{0}, t\right]$ be such that we have $\|u(\cdot, \tau)\|_{L^{q}(\mathbb{R})}>\lambda_{q}(t)$ for all $t_{0} \leq \tau<t_{2}$, while $\left\|u\left(\cdot, t_{2}\right)\right\|_{L^{q}(\mathbb{R})}=\lambda_{q}(t)$. We claim that $\|u(\cdot, \tau)\|_{L^{q}(\mathbb{R})} \leq \lambda_{q}(t)$ for every $t_{2} \leq \tau \leq t$ : in fact, if this were not true, we could then find $t_{3}, t_{4}$ with $t_{2} \leq t_{3}<t_{4} \leq t$ such that $\|u(\cdot, \tau)\|_{L^{q}(\mathbb{R})}>\lambda_{q}(t)$ for all $t_{3}<\tau \leq t_{4},\left\|u\left(\cdot, t_{3}\right)\right\|_{L^{q}(\mathbb{R})}=\lambda_{q}(t)$. By (20a), Theorem 2 , this would require $(d / d \tau)\|u(\cdot, \tau)\|_{L^{q}(\mathbb{R})}^{q}<0$ for all $\tau \in$ ]$\left.t_{3}, t_{4}\right] \backslash E_{q}$, so that $\|u(\cdot, \tau)\|_{L^{q}(\mathbb{R})}$ could not increase anywhere on $\left[t_{3}, t_{4}\right]$. This contradicts $\left\|u\left(\cdot, t_{3}\right)\right\|_{L^{q}(\mathbb{R})}<\left\|u\left(\cdot, t_{4}\right)\right\|_{L^{q}(\mathbb{R})}$, and so we have $\|u(\cdot, \tau)\|_{L^{q}(\mathbb{R})} \leq \lambda_{q}(t)$ for every $t_{2} \leq \tau \leq t$, as claimed. On the other hand, by $(20 \mathrm{a}),\|u(\cdot, \tau)\|_{L^{q}(\mathbb{R})}$ has to be monotonically decreasing on $\left[t_{0}, t_{2}\right]$, just as in Case I. Therefore, we have $\mathbb{U}_{q}\left(t_{0} ; t\right)=\left\|u\left(\cdot, t_{0}\right)\right\|_{L^{q}(\mathbb{R})}$ in this case again, which shows (26).

Case III. Consider $\left\|u\left(\cdot, t_{0}\right)\right\|_{L^{q}(\mathbb{R})} \leq \lambda_{q}(t)$. This gives $\|u(\cdot, \tau)\|_{L^{q}(\mathbb{R})} \leq \lambda_{q}(t)$ for every $t_{0} \leq \tau \leq t$, by repeating the argument used on the interval $\left[t_{2}, t\right]$ in Case II. It follows that we must have $\mathbb{U}_{q}\left(t_{0} ; t\right) \leq \lambda_{q}(t)$ in this case, and the proof of Theorem 3 is complete.

An important application of Theorem 3 is the following result.

Theorem 4. Let $p_{0} \leq p<\infty, 0 \leq t_{0}<T_{*}$. Then

$$
\begin{aligned}
& \|u(\cdot, t)\|_{L^{\infty}(\mathbb{R})} \\
& \quad \leq(2 p)^{1 / p} \cdot \max \left\{\left\|u\left(\cdot, t_{0}\right)\right\|_{L^{\infty}(\mathbb{R})} ; \mathbb{B}\left(t_{0} ; t\right)^{1 / p} \mathbb{U}_{p}\left(t_{0} ; t\right)\right\}
\end{aligned}
$$

for any $t_{0} \leq t<T_{*}$, where $\mathbb{B}\left(t_{0} ; t\right)$ and $\mathbb{U}_{p}\left(t_{0} ; t\right)$ are given in (24) and (25) above.

Proof. Let $k \in \mathbb{Z}, k \geq 2$. Applying (26) successively with $q=$ $2 p, 4 p, \ldots, 2^{k} p$, we obtain

$$
\begin{gathered}
\|u(\cdot, t)\|_{L^{2^{k} p(\mathbb{R})}} \\
\leq \max \left\{\left\|\mathbf{u}\left(\cdot, t_{0}\right)\right\|_{L^{2^{k} p(\mathbb{R})}} ; K(k, \ell)^{1 / p} \cdot \mathbb{B}\left(t_{0} ; t\right)^{(1 / p)\left(2^{-\ell}-2^{-k}\right)}\right. \\
\cdot\left\|u\left(\cdot, t_{0}\right)\right\|_{L^{2^{\ell} p(\mathbb{R})}}, 1 \leq \ell \leq k-1 ; \\
\left.K(k, 0)^{1 / p} \cdot \mathbb{B}\left(t_{0} ; t\right)^{(1 / p)\left(1-2^{-k}\right)} \cdot \mathbb{U}_{p}\left(t_{0} ; t\right)\right\},
\end{gathered}
$$

where

$$
K(k, \ell)=\prod_{j=\ell+1}^{k}\left(2^{j-1} p C_{2}^{3}\right)^{2^{-j}}, \quad 0 \leq \ell \leq k-1 .
$$

Now, for $1 \leq \ell \leq k-1$,

$$
\begin{gathered}
\mathbb{B}\left(t_{0} ; t\right)^{(1 / p)\left(2^{-\ell}-2^{-k}\right)} \cdot\left\|u\left(\cdot, t_{0}\right)\right\|_{L^{2^{\ell}} p(\mathbb{R})} \\
\leq \mathbb{B}\left(t_{0} ; t\right)^{(1 / p)\left(2^{-\ell}-2^{-k}\right)} \cdot\left\|u\left(\cdot, t_{0}\right)\right\|_{L^{p}(\mathbb{R})}^{\left(2^{-\ell}-2^{-k}\right) /\left(1-2^{-k}\right)} \\
\cdot\left\|u\left(\cdot, t_{0}\right)\right\|_{L^{2^{k} p(\mathbb{R})}}^{\left(1-2^{-\ell}\right) /\left(1-2^{-k}\right)} \\
\leq \max \left\{\left\|u\left(\cdot, t_{0}\right)\right\|_{L^{2^{k} p(\mathbb{R})}} ;\right. \\
\left.\mathbb{B}\left(t_{0} ; t\right)^{(1 / p)\left(1-2^{-k}\right)} \cdot\left\|u\left(\cdot, t_{0}\right)\right\|_{L^{p}(\mathbb{R})}\right\}
\end{gathered}
$$


by Young's inequality (see, e.g., [11, page 622]); in particular, we get, from (28a) and (28b),

$$
\begin{aligned}
& \|u(\cdot, t)\|_{L^{2^{k} p(\mathbb{R})}} \\
& \leq(2 p)^{1 / p} \\
& \quad \cdot \max \left\{\left\|u\left(\cdot, t_{0}\right)\right\|_{L^{2^{k} p(\mathbb{R})}} ; \mathbb{B}\left(t_{0} ; t\right)^{(1 / p)\left(1-2^{-k}\right)} \cdot \mathbb{U}_{p}\left(t_{0} ; t\right)\right\},
\end{aligned}
$$

since $K(k, \ell) \leq 2 p$ for all $0 \leq \ell \leq k-1$. Letting $k \rightarrow \infty$, (27) is obtained.

It follows from Theorems 1 and 4 that $u(\cdot, t)$ is globally defined $\left(T_{*}=\infty\right)$. Now, from (27), we immediately obtain, letting $t \rightarrow \infty$,

$$
\begin{aligned}
& \limsup _{t \rightarrow \infty}\|u(\cdot, t)\|_{L^{\infty}(\mathbb{R})} \\
& \quad \leq(2 p)^{1 / p} \cdot \max \left\{\left\|u\left(\cdot, t_{0}\right)\right\|_{L^{\infty}(\mathbb{R})} ; \mathbb{B}\left(t_{0}\right)^{1 / p} \mathbb{U}_{p}\left(t_{0}\right)\right\}
\end{aligned}
$$

for any $t_{0} \geq 0$, where $\mathbb{B}\left(t_{0}\right)$ and $\mathbb{U}_{p}\left(t_{0}\right)$ are given by

$$
\begin{gathered}
\mathbb{B}\left(t_{0}\right)=\sup \left\{B(t): t \geq t_{0}\right\}, \\
\mathbb{U}_{p}\left(t_{0}\right)=\sup \left\{\|u(\cdot, t)\|_{L^{p}(\mathbb{R})}: t \geq t_{0}\right\} .
\end{gathered}
$$

Taking $\left(t_{0}^{(n)}\right)_{n}$ such that $t_{0}^{(n)} \rightarrow \infty$ and $\left\|u\left(\cdot, t_{0}^{(n)}\right)\right\|_{L^{\infty}(\mathbb{R})} \rightarrow$ $\liminf \operatorname{in}_{t \rightarrow \infty}\|u(\cdot, t)\|_{L^{\infty}(\mathbb{R})}$, and applying (31) with $t_{0}=t_{0}^{(n)}$ for each $n$, we then obtain, letting $n \rightarrow \infty$,

$$
\begin{aligned}
& \underset{t \rightarrow \infty}{\limsup }\|u(\cdot, t)\|_{L^{\infty}(\mathbb{R})} \\
& \quad \leq(2 p)^{1 / p} \cdot \max \left\{\liminf _{t \rightarrow \infty}\|u(\cdot, t)\|_{L^{\infty}(\mathbb{R})} ; \mathscr{B}^{1 / p} \cdot \mathscr{U}_{p}\right\},
\end{aligned}
$$

where $\mathscr{B}$ and $\mathcal{U}_{p}$ are given by

$$
\mathscr{B}=\limsup _{t \rightarrow \infty} B(t), \quad \mathcal{U}_{p}=\limsup _{t \rightarrow \infty}\|u(\cdot, t)\|_{L^{p}(\mathbb{R})} .
$$

\section{Large Time Estimates}

In this section, we use the results obtained above to derive two basic large time estimates (given in Theorems 5 and 6) for solutions $u(\cdot, t)$ of problem (1a), (1b), which represent important intermediate steps that will ultimately lead to the main result stated in Theorem 7 .

Theorem 5. Let $q \geq 2 p_{0}$, and $\mathscr{B} \geq 0$ be as defined in (35). Then

$$
\begin{aligned}
& \limsup _{t \rightarrow \infty}\|u(\cdot, t)\|_{L^{q}(\mathbb{R})}, \\
& \quad \leq\left(\frac{q}{2} C_{2}^{3}\right)^{1 / q} \cdot \mathscr{B}^{1 / q} \cdot \underset{t \rightarrow \infty}{\limsup }\|u(\cdot, t)\|_{L^{q / 2}(\mathbb{R})},
\end{aligned}
$$

where $C_{2}=(3 \sqrt{3} /(4 \pi))^{1 / 3}$ is the constant in the Nash inequality (19).
Proof. We set $p=q / 2$ and assume that $\mathcal{U}_{p}$ is finite. As in the proof of Theorem 2 , we take $v \in L^{\infty}(\mathbb{R} \times[0, \infty[)$ given by $v(x, t)=|u(x, t)|^{p}$ if $p>1, v(x, t)=u(x, t)$ if $p=1$. It follows that

$$
\begin{gathered}
\|v(\cdot, t)\|_{L^{2}(\mathbb{R})}^{2}=\|u(\cdot, t)\|_{L^{2 p}(\mathbb{R})}^{2 p}, \\
\left\|v_{x}(\cdot, t)\right\|_{L^{2}(\mathbb{R})}^{2}=p^{2} \int_{\mathbb{R}}|u(x, t)|^{2 p-2}\left|u_{x}(x, t)\right|^{2} d x .
\end{gathered}
$$

Therefore, from (18), we have, for some null set $E_{2 p} \subset[0, \infty[$,

$$
\begin{aligned}
& \frac{d}{d t}\|v(\cdot, t)\|_{L^{2}(\mathbb{R})}^{2}+4\left(1-\frac{1}{2 p}\right)\left\|v_{x}(\cdot, t)\right\|_{L^{2}(\mathbb{R})}^{2} \\
& \quad \leq 4 p\left(1-\frac{1}{2 p}\right) B(t)\|v(\cdot, t)\|_{L^{2}(\mathbb{R})}\left\|v_{x}(\cdot, t)\right\|_{L^{2}(\mathbb{R})}
\end{aligned}
$$

for all $t \in\left[0, \infty\left[\backslash E_{2 p}\right.\right.$, and so, by (19),

$$
\begin{aligned}
& \frac{d}{d t}\|v(\cdot, t)\|_{L^{2}(\mathbb{R})}^{2}+4\left(1-\frac{1}{2 p}\right)\left\|v_{x}(\cdot, t)\right\|_{L^{2}(\mathbb{R})}^{2} \\
& \quad \leq 4 p C_{2}\left(1-\frac{1}{2 p}\right) B(t)\|v(\cdot, t)\|_{L^{1}(\mathbb{R})}^{2 / 3}\left\|v_{x}(\cdot, t)\right\|_{L^{2}(\mathbb{R})}^{4 / 3}
\end{aligned}
$$

This gives, by Young's inequality ([11, page 622]), for all $t \in$ $\left[0, \infty\left[\backslash E_{2 p}\right.\right.$,

$$
\begin{gathered}
\frac{d}{d t}\|v(\cdot, t)\|_{L^{2}(\mathbb{R})}^{2}+\frac{4}{3}\left(1-\frac{1}{2 p}\right)\left\|v_{x}(\cdot, t)\right\|_{L^{2}(\mathbb{R})}^{2} \\
\leq \frac{4}{3}\left(1-\frac{1}{2 p}\right)\left(p C_{2}\right)^{3} B(t)^{3}\|v(\cdot, t)\|_{L^{1}(\mathbb{R})}^{2} .
\end{gathered}
$$

Setting

$$
\begin{aligned}
\lambda_{p} & =\limsup _{t \rightarrow \infty} g(t), \\
g(t) & =\left(p C_{2}^{3}\right)^{1 / 2} B(t)^{1 / 2}\|v(\cdot, t)\|_{L^{1}(\mathbb{R})},
\end{aligned}
$$

we claim that

$$
\limsup _{t \rightarrow \infty}\|v(\cdot, t)\|_{L^{2}(\mathbb{R})} \leq \lambda_{p}
$$

In fact, let us argue by contradiction. If (42) is false, we can pick $0<\eta \ll 1$ and a sequence $\left(t_{j}\right)_{j \geq 0}, t_{j} \rightarrow \infty$, such that $\left\|v\left(\cdot, t_{j}\right)\right\|_{L^{2}(\mathbb{R})}>\lambda_{p}+\eta$ (for all $j \geq 0$ ) and $g(t) \leq \lambda_{p}+\eta / 2$ for all $t \geq t_{0}$. From (20a), Theorem 2 , it will then follow that

$$
\|v(\cdot, t)\|_{L^{2}(\mathbb{R})}>\lambda_{p}+\eta, \quad \forall t \geq t_{0} .
$$

In fact, suppose that (43) were false, so that we had $\|v(\cdot, \widetilde{t})\|_{L^{2}(\mathbb{R})} \leq \lambda_{p}+\eta$ for some $\tilde{t}>t_{0}$. Taking $j \gg 1$ with $t_{j}>\widetilde{t}$, we could then find $\hat{t} \in\left[\widetilde{t}, t_{j}\left[\right.\right.$ such that $\|v(\cdot, t)\|_{L^{2}(\mathbb{R})}>\lambda_{p}+\eta$ for all $\left.t \in] \widehat{t}, t_{j}\right]$, while $\|v(\cdot, \widehat{t})\|_{L^{2}(\mathbb{R})}=\lambda_{p}+\eta$, and so there would exist $t_{*} \in\left[\widehat{t}, t_{j}\right] \backslash E_{2 p}$ with $(d / d \tau)\|v(\cdot, t)\|_{L^{2}(\mathbb{R})}^{2}$ positive at $t=t_{*}$. By (20a), we would have $\left\|v\left(\cdot, t_{*}\right)\right\|_{L^{2}(\mathbb{R})} \leq \lambda_{p}$, but this would contradict the fact that $\|v(\cdot, t)\|_{L^{2}(\mathbb{R})} \geq \lambda_{p}+\eta$ 
everywhere on $\left[\widehat{t}, t_{j}\right]$. Thus, we conclude that (43) cannot be false, as claimed. We then obtain, from (19), (40), and (43),

$$
\begin{aligned}
& \|v(\cdot, t)\|_{L^{2}(\mathbb{R})}^{6} \\
& \quad \leq C_{2}^{6}\|v(\cdot, t)\|_{L^{1}(\mathbb{R})}^{4}\left\|v_{x}(\cdot, t)\right\|_{L^{2}(\mathbb{R})}^{2} \\
& \quad \leq g(t)^{6}+\frac{2 p}{2 p-1}\|v(\cdot, t)\|_{L^{1}(\mathbb{R})}^{4}\left(-\frac{d}{d t}\|v(\cdot, t)\|_{L^{2}(\mathbb{R})}^{2}\right)
\end{aligned}
$$

for all $t \in\left[t_{0}, \infty\left[\backslash E_{2 p}\right.\right.$. Recalling that $\|v(\cdot, t)\|_{L^{2}(\mathbb{R})}>\lambda_{p}+\eta$, $g(t) \leq \lambda_{p}+\eta / 2$, for all $t \geq t_{0}$, this gives

$$
-\frac{d}{d t}\|v(\cdot, t)\|_{L^{2}(\mathbb{R})}^{2} \geq K(\eta), \quad \forall t \in\left[t_{0}, \infty\left[\backslash E_{2 p}\right.\right.
$$

for some constant $K(\eta)>0$ independent of $t$, which cannot be, since this implies

$$
\left\|v\left(\cdot, t_{0}\right)\right\|_{L^{2}(\mathbb{R})}^{2} \geq K(\eta) \cdot\left(t-t_{0}\right) \quad \forall t>t_{0}
$$

This contradiction shows (42), which is equivalent to (36), and the proof is complete. get

Applying (36) successively with $q=2 p, 4 p, \ldots, 2^{k} p$, we

$$
\begin{aligned}
& \limsup _{t \rightarrow \infty}\|u(\cdot, t)\|_{L^{2^{k}} p(\mathbb{R})} \\
& \quad \leq\left[\prod_{j=1}^{k}\left(2^{j-1} p C_{2}^{3}\right)^{2^{-j}}\right]^{1 / p} \cdot \mathscr{B}^{(1 / p)\left(1-2^{-k}\right)} \cdot \mathscr{U}_{p}
\end{aligned}
$$

for $k \geq 1$ arbitrary, where $\mathcal{U}_{p}=\lim \sup _{t \rightarrow \infty}\|u(\cdot, t)\|_{L^{p}(\mathbb{R})}$. Letting $k \rightarrow \infty$, this suggests

$$
\limsup _{t \rightarrow \infty}\|u(\cdot, t)\|_{L^{\infty}(\mathbb{R})} \leq K(p) \cdot \mathscr{B}^{1 / p} \cdot \limsup _{t \rightarrow \infty}\|u(\cdot, t)\|_{L^{p}(\mathbb{R})},
$$

where

$$
K(p)=\left[\prod_{j=1}^{\infty}\left(2^{j-1} p C_{2}^{3}\right)^{2^{-j}}\right]^{1 / p}=\left(\frac{3 \sqrt{3}}{2 \pi} p\right)^{1 / p}
$$

(cf. (6) above), as long as the limit processes $k \rightarrow \infty$, $t \rightarrow \infty$ can be interchanged. That this is indeed the case is a consequence of (34) and the following result.

Theorem 6. Let $p \geq p_{0}$. Then

$$
\begin{aligned}
& \liminf _{t \rightarrow \infty}\|u(\cdot, t)\|_{L^{\infty}(\mathbb{R})} \\
& \quad \leq\left(p C_{2} C_{\infty}\right)^{1 / p} \cdot \mathscr{B}^{1 / p} \cdot \limsup _{t \rightarrow \infty}\|u(\cdot, t)\|_{L^{p}(\mathbb{R})},
\end{aligned}
$$

where $C_{2}, C_{\infty}$ are the constants given in (19) and (9).
Proof. Again, assuming $\mathcal{U}_{p}$ finite (otherwise, (49) is obvious; cf. endnote $\left.{ }^{4}\right)$, we introduce, as in the previous proof, $v \in$ $L^{\infty}\left(\mathbb{R} \times\left[0, \infty[)\right.\right.$ given by $v(x, t)=|u(x, t)|^{p}$ if $p>1$, and $v(x, t)=u(x, t)$ if $p=1$. Thus, (40) is valid, and setting $\lambda_{p} \in \mathbb{R}, g \in L^{\infty}([0, \infty[)$ by

$$
\lambda_{p}=\limsup _{t \rightarrow \infty} g(t), \quad g(t)=p C_{2} B(t)\|\mathbf{v}(\cdot, t)\|_{L^{1}(\mathbb{R})},
$$

we have that (49) is obtained if we show that

$$
\liminf _{t \rightarrow \infty}\|v(\cdot, t)\|_{L^{\infty}(\mathbb{R})} \leq C_{\infty} \cdot \lambda_{p} .
$$

We argue by contradiction and assume that (51) is false. Taking then $0<\eta \ll 1, t_{0} \gg 1$ so that $\|v(\cdot, t)\|_{L^{\infty}(\mathbb{R})} \geq$ $C_{\infty} \cdot\left(\lambda_{p}+\eta\right)$ and $g(t) \leq \lambda_{p}+\eta / 2$ hold for all $t \geq t_{0}$, we get, by (9) and (40),

$$
\begin{aligned}
\|v(\cdot, t)\|_{L^{\infty}(\mathbb{R})}^{3} \leq & C_{\infty}^{3}\|v(\cdot, t)\|_{L^{1}(\mathbb{R})}\left\|v_{x}(\cdot, t)\right\|_{L^{2}(\mathbb{R})}^{2} \\
\leq & C_{\infty}^{3} g(t)^{3}+C_{\infty}^{3} \frac{2 p}{2 p-1}\|v(\cdot, t)\|_{L^{1}(\mathbb{R})} \\
& \times\left(-\frac{d}{d t}\|v(\cdot, t)\|_{L^{2}(\mathbb{R})}^{2}\right)
\end{aligned}
$$

for all $t \in\left[t_{0}, \infty\left[\backslash E_{2 p}\right.\right.$. Since $\|v(\cdot, t)\|_{L^{\infty}(\mathbb{R})} \geq C_{\infty} \cdot\left(\lambda_{p}+\eta\right)$, $g(t) \leq \lambda_{p}+\eta / 2$, this gives

$$
-\frac{d}{d t}\|v(\cdot, t)\|_{L^{2}(\mathbb{R})}^{2} \geq K(\eta), \quad \forall t \in\left[t_{0}, \infty\left[\backslash E_{2 p}\right.\right.
$$

for some constant $K(\eta)>0$ independent of $t$. As before, this implies that $\left\|v\left(\cdot, t_{0}\right)\right\|_{L^{2}(\mathbb{R})}^{2} \geq K(\eta) \cdot\left(t-t_{0}\right)$ for all $t \geq$ $t_{0}$, which is impossible because $\left\|v\left(\cdot, t_{0}\right)\right\|_{L^{2}(\mathbb{R})}$ is finite. This contradiction establishes (51) above, completing the proof of Theorem 6 .

We are finally in good position to derive (6), (48a), and (48b). Combining (34) and (49) above, we obtain

$$
\limsup _{t \rightarrow \infty}\|u(\cdot, t)\|_{L^{\infty}(\mathbb{R})} \leq\left(2 p^{2}\right)^{1 / p} \cdot \mathscr{B}^{1 / p} \cdot \mathcal{U}_{p}
$$

for each $p \geq p_{0}$, so that we have, in particular,

$$
\limsup _{t \rightarrow \infty}\|u(\cdot, t)\|_{L^{\infty}(\mathbb{R})} \leq\left(2^{2 k+1} p^{2}\right)^{1 / 2^{k} p} \cdot \mathscr{B}^{1 / 2^{k} p} \cdot \mathscr{U}_{2^{k} p}
$$

for each $k \geq 0$. By (47), we then get

$$
\begin{aligned}
& \limsup _{t \rightarrow \infty}\|u(\cdot, t)\|_{L^{\infty}(\mathbb{R})} \\
& \quad \leq\left\{\left(2^{2 k+1} p^{2}\right)^{2^{-k}} \cdot \prod_{j=1}^{k}\left(2^{j-1} p C_{2}^{3}\right)^{2^{-j}}\right\}^{1 / p} \cdot \mathscr{B}^{1 / p} \cdot \mathscr{U}_{p}
\end{aligned}
$$

for all $k$. Letting $k \rightarrow \infty$, Theorem 7 is obtained, and our argument is complete. 
Theorem 7. Let $p \geq p_{0}$. Assuming $b \in L^{\infty}(\mathbb{R} \times[0, \infty[)$, then (6), (48a), and (48b) hold.

It is worth noticing that the corresponding estimate for the $n$-dimensional problem (8), namely,

$$
\begin{aligned}
& \limsup _{t \rightarrow \infty}\|u(\cdot, t)\|_{L^{\infty}\left(\mathbb{R}^{n}\right)} \\
& \quad \leq K(n, p) \cdot \mathscr{B}^{n / p} \cdot \limsup _{t \rightarrow \infty}\|u(\cdot, t)\|_{L^{p}\left(\mathbb{R}^{n}\right)},
\end{aligned}
$$

where $\mathscr{B} \geq 0$ is similarly defined, can be also derived in arbitrary dimension $n>1$.

\section{Concluding Remarks}

We close our discussion of the problem (1a), (1b), given $b \in$ $L^{\infty}\left(\mathbb{R} \times\left[0, \infty[), 1 \leq p_{0}<\infty\right.\right.$, indicating a few questions which were not answered by our analysis:

(a) characterize all $b \in L^{\infty}(\mathbb{R} \times[0, \infty[)$ for which it is true that $\|u(\cdot, t)\|_{L^{\infty}(\mathbb{R})} \rightarrow 0$ (as $t \rightarrow \infty$ ) for every solution $u(\cdot, t)$ of problem (1a) and (1b);

(b) same question as (a) above, but requiring only that limsup $\|u(\cdot, t)\|_{L^{\infty}(\mathbb{R})}<\infty$ (as $\left.t \rightarrow \infty\right)$ for every solution $u(\cdot, t)$ of problem (1a) and (1b), in case $p_{0}>$ $1 ;^{5}$

(c) given $p_{0}>1$, characterize all $b \in L^{\infty}(\mathbb{R} \times[0, \infty[)$ such that $\|u(\cdot, t)\|_{L^{p_{0}(\mathbb{R})}} \rightarrow 0($ as $t \rightarrow \infty)$ for every solution $u(\cdot, t)$ of problem (1a) and (1b);

(d) same question as (c) above, but requiring only that $\lim \sup \|u(\cdot, t)\|_{L^{p_{0}(\mathbb{R})}}<\infty$ (as $\left.t \rightarrow \infty\right)$ for every solution $u(\cdot, t)$ of problem (1a) and (1b);

(e) for $p_{0}=1$, characterize all $b \in L^{\infty}(\mathbb{R} \times[0, \infty[)$ such that $\|u(\cdot, t)\|_{L^{1}(\mathbb{R})} \rightarrow|m|($ as $t \rightarrow \infty)$ for every solution $u(\cdot, t)$, where $m=\int_{\mathbb{R}} u_{0}(x) d x$ is the solution mass;

(f) for $p_{0}=1$, and $b \in L^{\infty}(\mathbb{R} \times[0, \infty[)$ not satisfying property (e), what are the values of $\lim _{t \rightarrow \infty}\|u(\cdot, t)\|_{L^{1}(\mathbb{R})}$ in case of initial states that change sign?

These questions can be similarly posed for solutions $u(\cdot, t)$ of autonomous problems

$$
u_{t}+(b(x) u)_{x}=u_{x x}, \quad u(\cdot, 0) \in L^{p_{0}}(\mathbb{R}) \cap L^{\infty}(\mathbb{R}),
$$

where $b \in L^{\infty}(\mathbb{R})$ does not depend on the time variable. For (58), question (e) has been answered in [12] (see also [13]). Another interesting question is the following:

(g) when (58) admits no stationary solutions other than the trivial solution $u=0$, is it true that $\lim _{t \rightarrow \infty}\|u(\cdot, t)\|_{L^{\infty}(\mathbb{R})}=0$ for every solution $u(\cdot, t)$ ?

Moreover, for solutions $u(\cdot, t)$ of (1a) and (1b) or (58) with $\|u(\cdot, t)\|_{L^{\infty}(\mathbb{R})} \rightarrow 0$ as $t \rightarrow \infty$, there is the question of determining the proper decay rate. ${ }^{6}$ As suggested by Figure 1 , solution decay may sometimes happen at remarkably slow rates.

\section{Conflict of Interests}

The authors declare that there is no conflict of interests regarding the publication of this paper.

\section{Acknowledgment}

The authors would like to thankCNPq (Conselho Nacional de Desenvolvimento Científico e Tecnológico, Brazil) for their financial support.

\section{Endnotes}

1. In (6), (11), and other similar expressions in the text, it is assumed that $0 \cdot \infty=\infty$.

2. The constants $(3 \sqrt{3} p /(2 \pi))^{1 / p}$ in (6) and (7) are not optimal; minimal values are not known.

3. In (6), (11), and other similar expressions in the text, it is assumed that $0 \cdot \infty=\infty$.

4. In (6), (11), and other similar expressions in the text, it is assumed that $0 \cdot \infty=\infty$.

5. For $p_{0}=1$, any $b \in L^{\infty}(\mathbb{R} \times[0, \infty[)$ satisfies property (b); compare (7) in Section 1.

6. In case we have $b_{x} \geq 0$ for all $x, t$, the answer is given in (2) above.

\section{References}

[1] C. J. Amick, J. L. Bona, and M. E. Schonbek, "Decay of solutions of some nonlinear wave equations," Journal of Differential Equations, vol. 81, no. 1, pp. 1-49, 1989.

[2] P. Braz e Silva, L. Schütz, and P. R. Zingano, "On some energy inequalities and supnorm estimates for advection-diffusion equations in $\mathbb{R}^{n}$," Nonlinear Analysis: Theory, Methods \& Applications, vol. 93, pp. 90-96, 2013.

[3] M. Escobedo and E. Zuazua, "Large time behavior for convection-diffusion equations in $\mathbb{R}^{n}$," Journal of Functional Analysis, vol. 100, no. 1, pp. 119-161, 1991.

[4] M. M. Porzio, "On decay estimates," Journal of Evolution Equations, vol. 9, no. 3, pp. 561-591, 2009.

[5] M. E. Schonbek, "Uniform decay rates for parabolic conservation laws," Nonlinear Analysis, vol. 10, no. 9, pp. 943-956, 1986.

[6] W. G. Melo, A priori estimates for various systems of advectiondiffusion equations (Portuguese) [Ph.D. thesis], Universidade Federal de Pernambuco, Recife, Brazil, 2011.

[7] L. S. Oliveira, Two results in classical analysis (Portuguese) [Ph.D. thesis], Graduate Program in Applied and Computational Mathematics (PPGMAp), Universidade Federal do Rio Grande do Sul, Porto Alegre, Brazil, 2013.

[8] D. Serre, Systems of Conservation Laws, vol. 1, Cambridge University Press, Cambridge, UK, 1999.

[9] J. Nash, "Continuity of solutions of parabolic and elliptic equations," American Journal of Mathematics, vol. 80, pp. 931954, 1958.

[10] E. A. Carlen and M. Loss, "Sharp constant in Nash's inequality," International Mathematics Research Notices, vol. 1993, pp. 213215, 1993. 
[11] L. C. Evans, Partial Differential Equations, American Mathematical Society, Providence, RI, USA, 2002.

[12] R. Rudnicki, "Asymptotical Stability in $L^{1}$ of Parabolic Equations," Journal of Differential Equations, vol. 102, no. 2, pp. 391401, 1993.

[13] Z. Brzeźniak and B. Szafirski, "Asymptotic behaviour of $L^{1}$ norm of solutions to parabolic equations," Bulletin of the Polish Academy of Sciences Mathematics, vol. 39, pp. 1-10, 1991. 


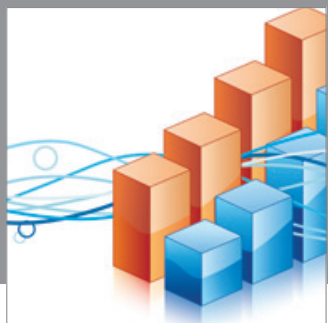

Advances in

Operations Research

mansans

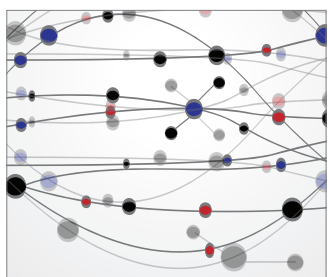

The Scientific World Journal
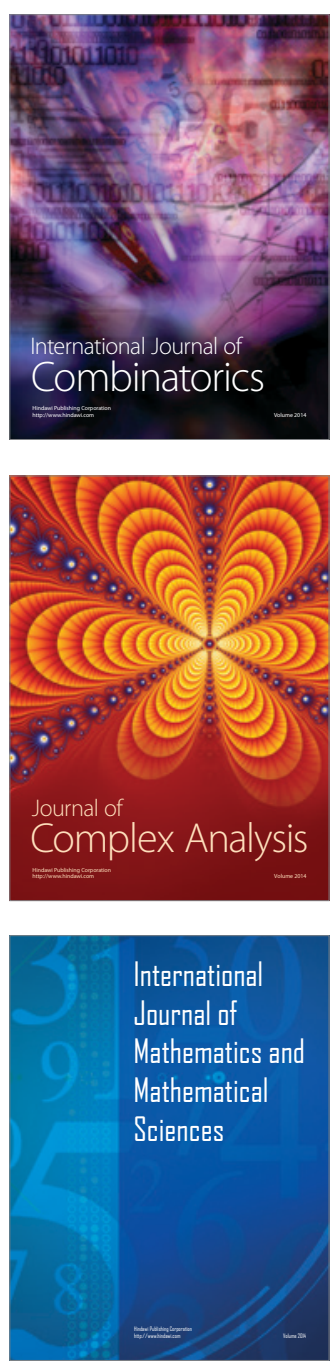
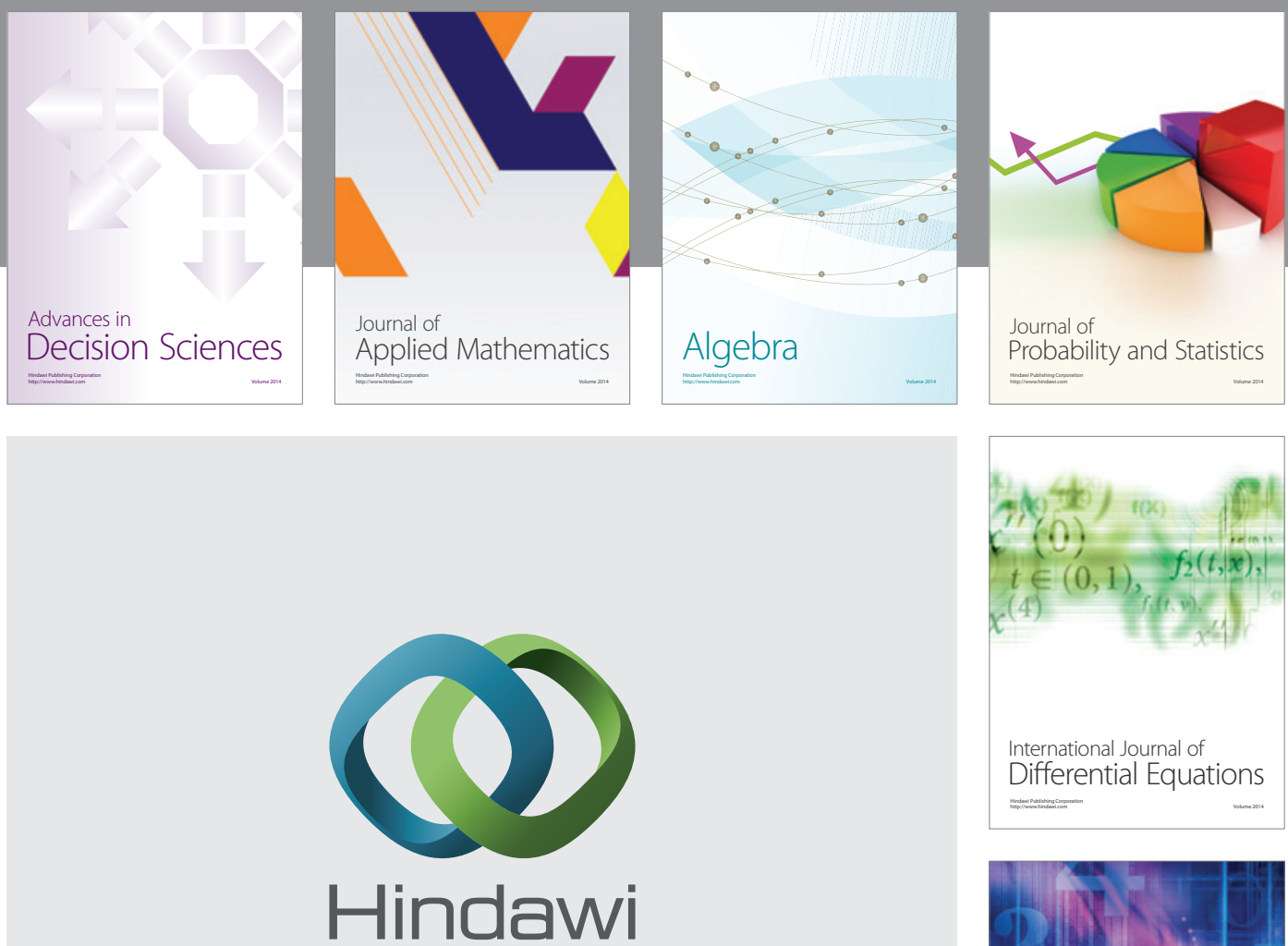

Submit your manuscripts at http://www.hindawi.com
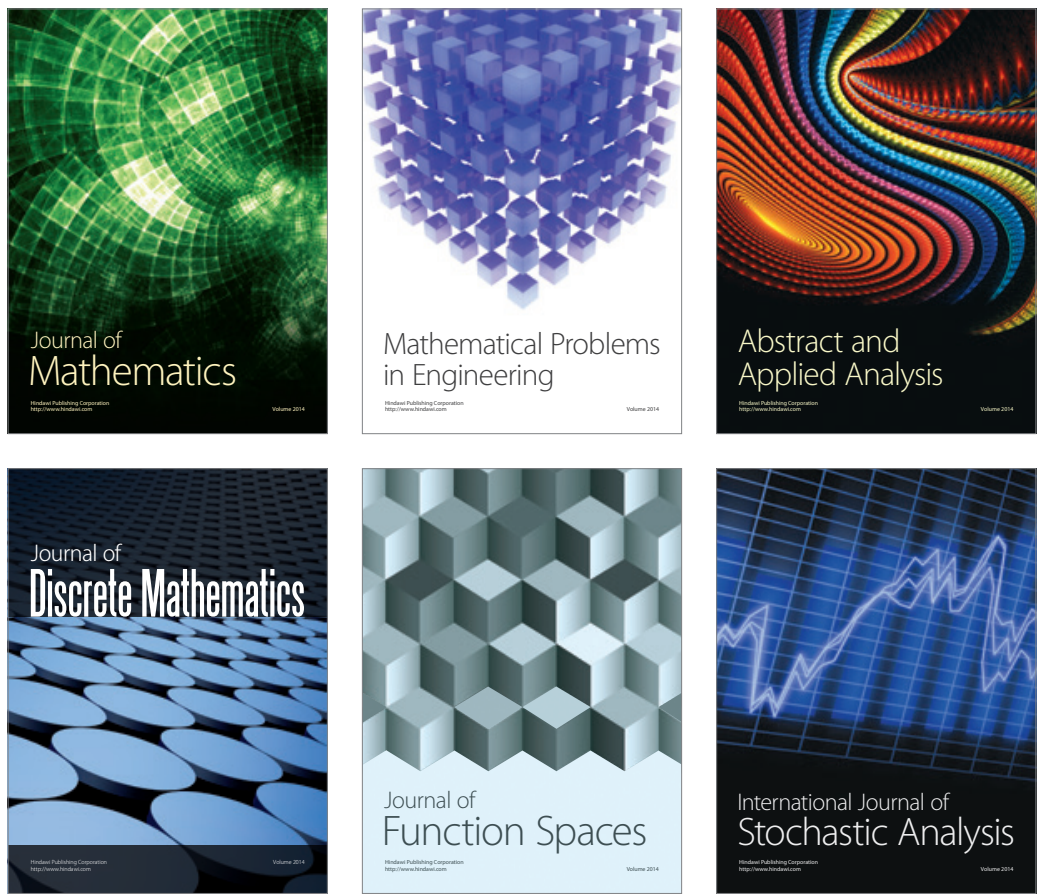

Journal of

Function Spaces

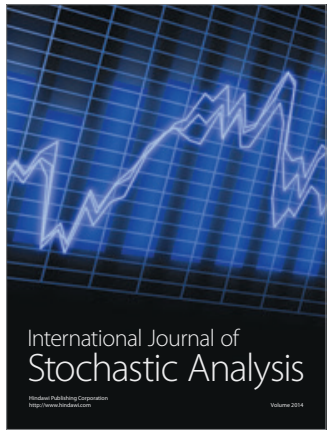

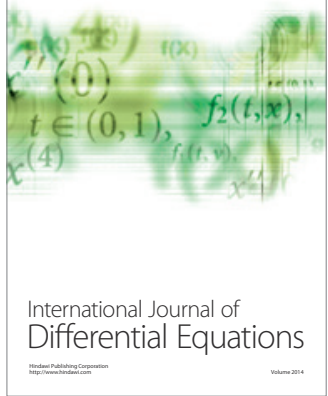
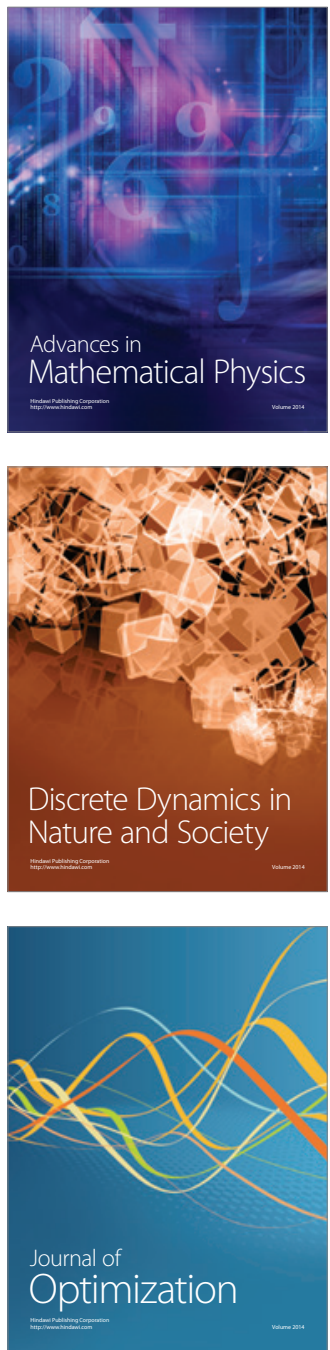